HEPHY-PUB 754/02

hep-ph/0204280

\title{
Radiative corrections to single Higgs boson production in $e^{+} e^{-}$annihilation
}

\author{
H. Eberl, W. Majerotto, and V. C. Spanos \\ Institut für Hochenergiephysik der Österreichischen Akademie der Wissenschaften, \\ A-1050 Vienna, Austria
}

\begin{abstract}
For energies relevant to future linear colliders, $\sqrt{s} \gtrsim 500 \mathrm{GeV}$, the $W W$ fusion channel dominates the Higgs boson production cross section $e^{+} e^{-} \rightarrow \bar{\nu} \nu h^{0}$. We have calculated the one-loop corrections to this process due to fermion and sfermion loops in the context of the MSSM. As a special case, the contribution of the fermion loops in the SM has also been studied. In general, the correction is negative and sizeable of the order of $10 \%$, the bulk of it being due to fermion loops.
\end{abstract}


As no Higgs boson could be detected so far, the search for the Higgs boson as the primary goal of high energy physics will continue. The four LEP experiments delivered a lower bound for the Standard Model (SM) Higgs mass, $m_{h} \gtrsim 114 \mathrm{GeV}$ [1]. In $e^{+} e^{-}$ collisions, for energies $\gtrsim 200 \mathrm{GeV}$, the production of a single Higgs boson plus missing

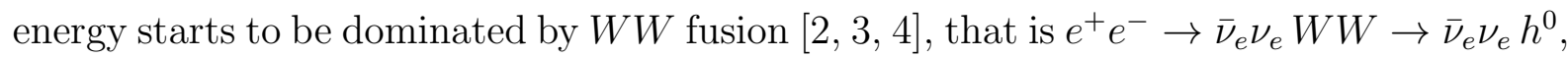
whereas the Higgsstrahlung process [5] $e^{+} e^{-} \rightarrow Z h^{0} \rightarrow \bar{\nu} \nu h^{0}$ becomes less important. $h^{0}$ denotes the SM Higgs boson or the light Higgs boson of the Minimal Supersymmetric Standard Model (MSSM), while $H^{0}$ is the heavy $C P$-even Higgs boson in the MSSM. The rates for the $Z Z$ fusion are generally one order of magnitude smaller than those of the $W W$ channel.

At LHC, in $p p$ collisions, the gluon-gluon fusion mechanism provides the dominant contribution to Higgs production. The next important Higgs production channel is vectorboson fusion $V V \rightarrow h^{0} / H^{0}$. In particular, it provides an additional event signature due to the two energetic forward jets. Recently, it has been argued that the channels $W W \rightarrow h^{0} / H^{0} \rightarrow \tau \bar{\tau}$ and $W W$, can serve as suitable search channels at LHC even for a Higgs boson mass of $m_{h} \sim 120 \mathrm{GeV}$ [6].

At Tevatron, with $p \bar{p}$ collisions at $2 \mathrm{TeV}$, the $W W$ fusion process plays a less important rôle. The $g g$ fusion is the dominant process for Higgs production there, but the Higgsstrahlung $q \bar{q} \rightarrow W \rightarrow W h^{0}$ is larger than the $W W$ fusion for $m_{h} \lesssim 180 \mathrm{GeV}$ [7].

It is worth mentioning that $W W$ (and $Z Z$ ) fusion is also the most important Higgs boson production mechanism in ep collisions. Actually, it was shown [8] that the epoption at LHC would offer the best opportunity to search for a Higgs boson in the mass range $m_{h}<140 \mathrm{GeV}$.

In this paper, we have calculated the leading one-loop corrections to the $W W h^{0}$ vertex in the MSSM by taking into account fermion/sfermion loops. They are supposed to be the dominant corrections due to the Yukawa couplings. We have applied them to the Higgs boson production in $e^{+} e^{-}$annihilation in the energy range $\sqrt{s}=0.5-3 \mathrm{TeV}$, i. e. $e^{+} e^{-} \rightarrow \bar{\nu}_{e} \nu_{e} W W \rightarrow \bar{\nu}_{e} \nu_{e} h^{0}$. We included the Higgsstrahlung process $e^{+} e^{-} \rightarrow Z h^{0} \rightarrow$ $\bar{\nu} \nu h^{0}$ and the interference between these two mechanisms. Because the Higgsstrahlung process is much smaller in this range, we have neglected its radiative corrections. We have also discussed the SM case where we have studied the dependence on the Higgs boson mass. As to the one-loop corrections to the $W W h^{0}$ vertex in the SM, we quite generally refer to the review article [9]. For this coupling also QCD corrections were included, the $\mathcal{O}\left(\alpha_{s} G_{F} m_{t}^{2}\right)$ in 10 and $\mathcal{O}\left(\alpha_{s}^{2} G_{F} m_{t}^{2}\right)$ in [1].

As for energies $\sqrt{s}>500 \mathrm{GeV}$ the dominant channel $e^{+} e^{-} \rightarrow \bar{\nu} \nu h^{0}$ is by far the $W W$ 


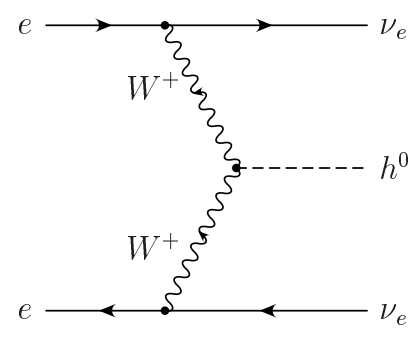

$\mathcal{M}_{W}^{\text {tree }}$

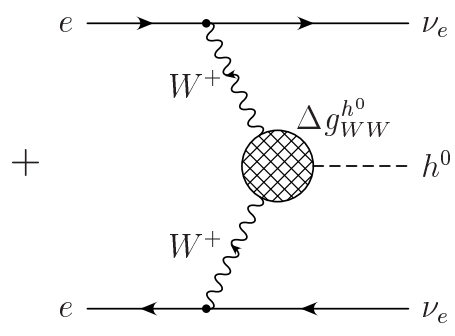

$\Delta \mathcal{M}_{W}$

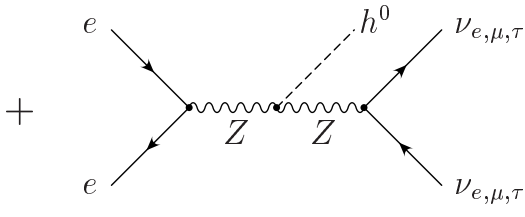

$\mathcal{M}_{Z}^{\text {tree }}$

Figure 1: The Feynman graphs for the process $e^{+} e^{-} \rightarrow \bar{\nu} \nu h^{0}$. Note that for $\left|\mathcal{M}_{Z}^{\text {tree }}\right|^{2}$ one has to sum over all three neutrino flavors.

fusion, in the context of our calculation only corrections to the $W W h^{0}$ vertex must be evaluated, see Fig. 1. At the one-loop level the Lagrangian for the $W W h^{0}$ coupling can be written as

$$
\mathcal{L}=\left(g_{W W}^{h^{0}} g^{\mu \nu}+\left(\Delta g_{W W}^{h^{0}}\right)^{\mu \nu}\right) h^{0} W_{\mu}^{+} W_{\nu}^{-},
$$

where the tree-level coupling in the MSSM can be read as $g_{W W}^{h^{0}}=g m_{W} \sin (\beta-\alpha)$. The one-loop part of the $W W h^{0}$ coupling in Eq. (1) can be expressed in terms of all possible form factors" as

$$
\left(\Delta g_{W W}^{h^{0}}\right)^{\mu \nu}=F^{00} g^{\mu \nu}+F^{11} k_{1}^{\mu} k_{1}^{\nu}+F^{22} k_{2}^{\mu} k_{2}^{\nu}+F^{12} k_{1}^{\mu} k_{2}^{\nu}+F^{21} k_{2}^{\mu} k_{1}^{\nu}+i F^{\epsilon} \epsilon^{\mu \nu \rho \delta} k_{1 \rho} k_{2 \delta},
$$

where $k_{1,2}$ denote the four-momenta of the off-shell $W$-bosons. At tree-level only the structure with $g_{\mu \nu}$ is present, and therefore all form factors but $F^{00}$ have to be ultra violet (UV) finite without being renormalized. By adding appropriate counter terms also the form factor $F^{00}$ is rendered UV finite. For the renormalization procedure the on-shell scheme has been adopted.

Actually, for the calculation of the one-loop corrected $W W h^{0}$ vertex we compute the vertex and the wave-function corrections stemming from the graphs of Fig. 2, as well as the coupling correction,

$$
\left(\Delta g_{W W}^{h^{0}}\right)^{\mu \nu}=\left(\delta g_{W W}^{h^{0}(v)}\right)^{\mu \nu}+\left(\delta g_{W W}^{h^{0}(w)}+\delta g_{W W}^{h^{0}(c)}\right) g^{\mu \nu} .
$$

Comparing Eq. (3) with Eq. (21), it is obvious that the counter terms, i. e. the wavefunction and coupling corrections, contribute only to $F^{00}$. The wave-function correction is

$$
\delta g_{W W}^{h^{0}(w)}=g_{W W}^{h^{0}}\left(\frac{1}{2}\left(\delta Z_{H}\right)_{h^{0} h^{0}}+\delta Z_{W}\right)+\frac{1}{2} g_{W W}^{H^{0}}\left(\delta Z_{H}\right)_{H^{0} h^{0}},
$$

\footnotetext{
${ }^{1}$ As the expressions for $F^{00}, F^{11}, \ldots, F^{\epsilon}$ are quite lengthy they will be presented elsewhere [12].
} 

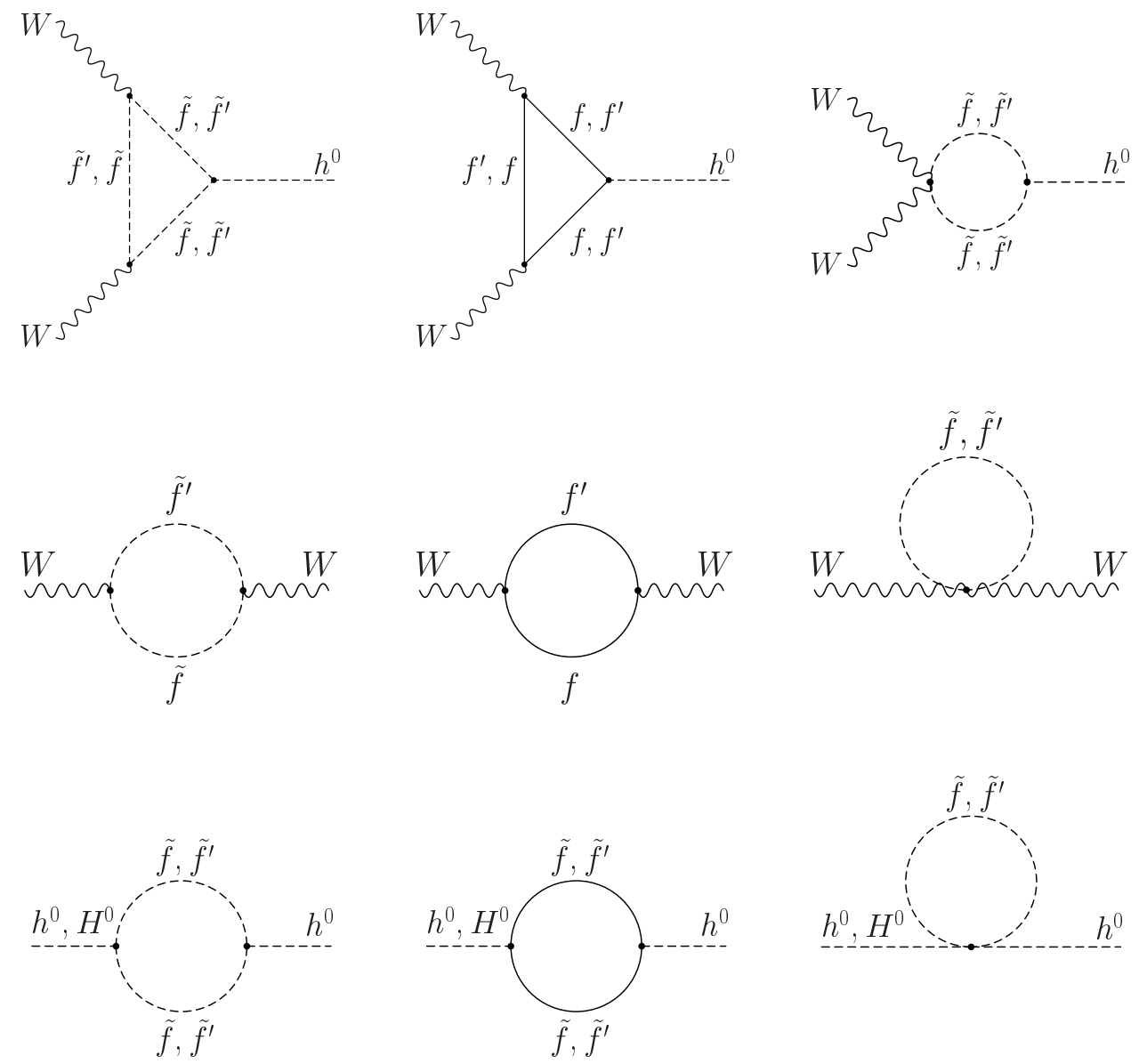

Figure 2: The Feynman graphs that contribute to the vertex corrections defined in Eq. (3) and the wave-function in Eq. (田). $f\left(f^{\prime}\right)$ denotes the up (down) type fermion.

where $\delta Z_{H}$ and $\delta Z_{W}$ are the symmetrized Higgs boson and the $W$-boson wave-function correction, respectively, calculated from the corresponding graphs of Fig. 2. In the case of the off-shell $W$-bosons, $\delta Z_{W}$ has the form

$$
\delta Z_{W}=\frac{\delta m_{W}^{2}-\Re \Pi_{W W}^{T}\left(k_{1}^{2}\right)}{k_{1}^{2}-m_{W}^{2}}+\frac{\delta m_{W}^{2}-\Re \Pi_{W W}^{T}\left(k_{2}^{2}\right)}{k_{2}^{2}-m_{W}^{2}}+2 \frac{\delta g}{g} .
$$

The coupling correction is

$$
\delta g_{W W}^{h^{0}(c)}=\left(\frac{\delta g}{g}+\frac{\delta m_{W}}{m_{W}}\right) g_{W W}^{h^{0}}+\frac{\sin 2 \beta}{2} \frac{\delta \tan \beta}{\tan \beta} g_{W W}^{H^{0}} .
$$

The expressions on the right-hand sides of Eqs. (14 6) can be found in Ref. [13]. Especially, we fixed the counter term $\delta \tan \beta$ by the on-shell condition $\operatorname{Im} \hat{\Pi}_{A Z}\left(m_{A}^{2}\right)=0$, where $\hat{\Pi}_{A Z}\left(m_{A}^{2}\right)$ is the renormalized self-energy for the mixing of the pseudo-scalar Higgs boson $A^{0}$ and $Z$-boson. 
Having calculated the form factors of Eq. (2), one can proceed to the calculation of the one-loop corrected cross section. The tree-level part of the amplitude was already calculated in Ref. 四. Here we will include also the one-loop correction, that is the interferences between the tree-level and the one-loop amplitudes of Fig. 进. For these terms we get

$$
2 \Re\left[\Delta \mathcal{M}_{W}\left(\mathcal{M}_{W}^{\text {tree }}\right)^{\dagger}\right]=4 g_{W W}^{h^{0}} g^{4}\left(2 F^{00} p_{1} \cdot p_{4} p_{2} \cdot p_{3}+F^{21} S\right) \prod_{i=1,2} \frac{1}{\left(k_{i}^{2}-m_{W}^{2}\right)^{2}}
$$

and

$$
\begin{aligned}
2 \Re\left[\Delta \mathcal{M}_{W}\left(\mathcal{M}_{Z}^{\text {tree }}\right)^{\dagger}\right] & =4 C_{L}^{e} g_{Z Z}^{h^{0}} \frac{g^{4}}{\cos ^{2} \theta_{W}}\left(2 F^{00} p_{1} \cdot p_{4} p_{2} \cdot p_{3}+F^{21} S\right) \\
& \times \frac{q_{2}^{2}-m_{Z}^{2}}{\left(q_{1}^{2}-m_{Z}^{2}\right)\left(\left(q_{2}^{2}-m_{Z}^{2}\right)^{2}+m_{Z}^{2} \Gamma_{Z}^{2}\right)} \prod_{i=1,2} \frac{1}{k_{i}^{2}-m_{W}^{2}},
\end{aligned}
$$

where

$$
\begin{aligned}
S & =\left(p_{1} \cdot p_{4}+p_{2} \cdot p_{3}\right)\left(p_{1} \cdot p_{2} p_{3} \cdot p_{4}+p_{1} \cdot p_{4} p_{2} \cdot p_{3}-p_{1} \cdot p_{3} p_{2} \cdot p_{4}\right) \\
& -2\left(p_{1} \cdot p_{2}+p_{3} \cdot p_{4}\right) p_{1} \cdot p_{4} p_{2} \cdot p_{3},
\end{aligned}
$$

$\Gamma_{Z}$ is the total $Z$-boson width, and $C_{L}^{e}=-\frac{1}{2}+\sin ^{2} \theta_{W}$. The kinematics has been chosen as $e^{-}\left(p_{1}\right) e^{+}\left(p_{2}\right) \rightarrow \nu_{e}\left(p_{3}\right) \bar{\nu}_{e}\left(p_{4}\right) h^{0}(p)$. We have also defined $k_{1}=p_{3}-p_{1}, k_{2}=p_{2}-p_{4}$, $q_{1}=p_{1}+p_{2}$ and $q_{2}=p_{3}+p_{4}$.

For the calculation of the cross section at tree-level, it is possible to perform some of the phase space integrations analytically and the rest of them numerically [3, 4]. However including the one-loop correction terms of Eq. (7) and (8), it is impossible to perform any of these integrations analytically. Therefore, we have performed the integrations numerically, using efficient numerical integration subroutines found in the NAG library. We have also checked that for the tree-level case our completely numerical calculation agrees with high accuracy with the semi-analytical results of Ref. [4.

Before embarking on discussing our numerical findings, we will make some comments concerning some details of our calculation. For the calculation of the supersymmetric (SUSY) Higgs boson masses and the Higgs mixing angle $\alpha$, a computer programme based on Ref. 14 has been used. The tree-level $W W h^{0}$ coupling for values of $\tan \beta>5$ as preferred by the LEP Higgs boson searches, mimics the SM one. For the calculation of

\footnotetext{
${ }^{2} \mathrm{As}$ it has already been mentioned the corresponding corrections to the Higgsstrahlung process can be safely ignored due to absolute dominance of the $W W$ fusion for $\sqrt{s}>500 \mathrm{GeV}$.
} 

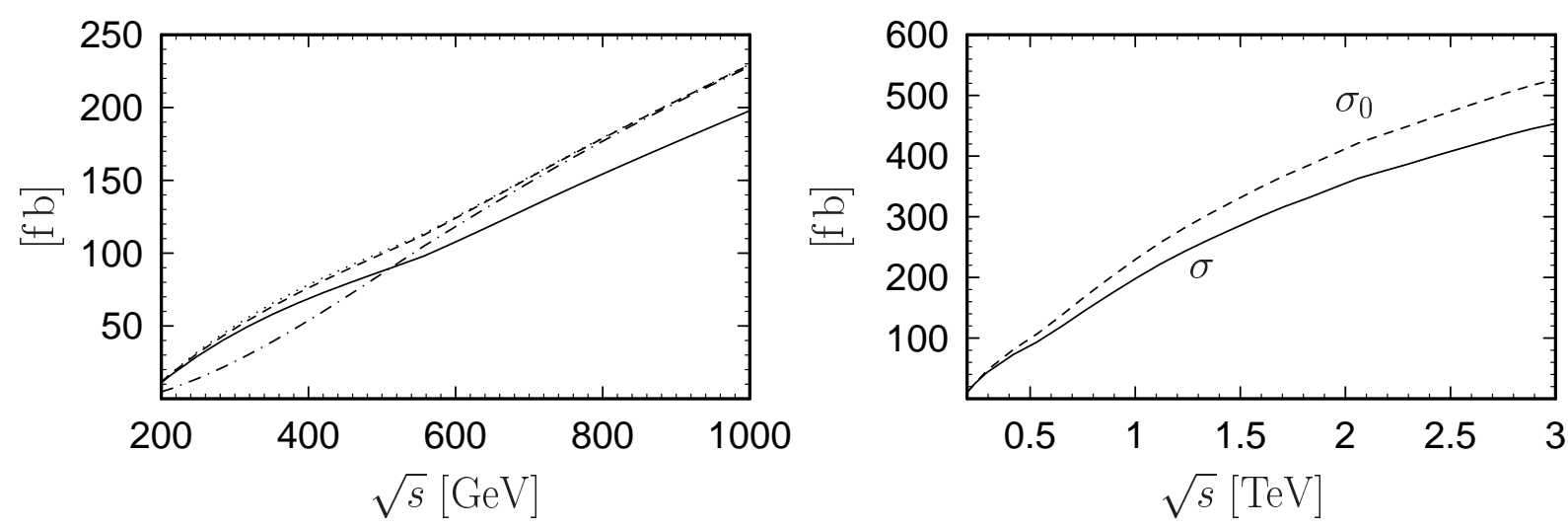

Figure 3: The various cross sections as functions of $\sqrt{s}$ (left). The dotted-dashed line represents the tree-level cross section $\sigma_{0}^{W W}$, the dotted line $\sigma_{0}^{W W}+\sigma_{0}^{h-s t r}$. The dashed line includes also the interference term $\sigma_{0}^{\text {interf. }}$ and represents the total tree-level cross section. The solid line includes the one-loop correction. The SUSY parameters are: $\tan \beta=10, \mu=-100 \mathrm{GeV}, A=-500 \mathrm{GeV}, m_{\tilde{Q}}=300 \mathrm{GeV}, M_{A}=500 \mathrm{GeV}$, and $M_{2}=400 \mathrm{GeV}$. For the same set of parameters also the tree-level cross section $\sigma_{0}$ and the one-loop corrected $\sigma$ are plotted for $\sqrt{s}$ up to $3 \mathrm{TeV}$ (right).

the fermion/sfermion one-loop corrections to the $W W h^{0}$ vertex, the contribution of the third family of fermions/sfermions has been taken into account. This contribution turns out to be the dominant one, in comparison with the first two families corrections, due to the large values of the Yukawa couplings $h_{t}$ and $h_{b}$. The effect of the running of the coupling constants $g$ and $g^{\prime}$ has been taken into account.

We now turn to the discussion of our numerical results. In Fig. 3 (left) we can see the various cross sections as a function of $\sqrt{s}$ for values up to $1 \mathrm{TeV}$. The dotted-dashed line represents the contribution from the $W W$ channel at tree-level alone, whereas the dotted line includes the Higgsstrahlung contribution as well. The dashed line comprises in addition the interference between the $W W$ channel and Higgsstrahlung. One can perceive that the size of this interference term is extremely small, and for this reason the difference between the dotted and dashed lines is rather minute. It is also clear that for $\sqrt{s} \gtrsim 500 \mathrm{GeV}$ the $W W$ fusion contribution dominates the total cross section for the Higgs production $e^{+} e^{-} \rightarrow \bar{\nu} \nu h^{0}$. Actually, for $\sqrt{s} \gtrsim 800 \mathrm{GeV}$ the total tree-level cross section is due to $W W$ fusion. In the solid line we have taken into account the one-loop correction from the fermion/sfermion loops. The correction is always negative with a significant size of the order of $10 \%$. This can also be seen from Fig. 3 (right), where we have plotted the tree-level cross section $\sigma_{0}$ (dashed line) and the one-loop 


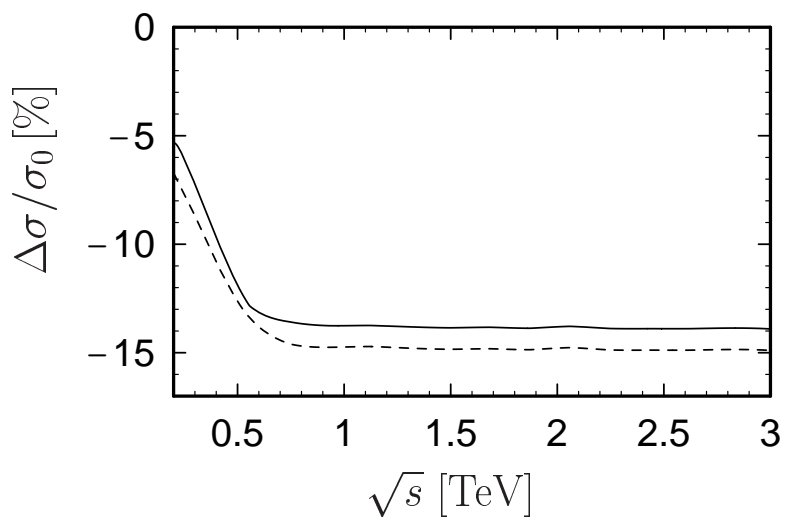

Figure 4: The relative correction $\Delta \sigma / \sigma_{0}$ as a function of $\sqrt{s}\left(\Delta \sigma=\sigma-\sigma_{0}\right)$, where $\sigma_{0}$ is the tree-level and $\sigma$ the one-loop corrected cross section. The solid and dashed lines correspond to two different choices of the SUSY parameters, as described in the text.

corrected cross section $\sigma$ (solid line) for energies up to $3 \mathrm{TeV}$. For simplicity, for all plots we have used $A_{t}=A_{b}=A_{\tau}=A,\left\{m_{\tilde{U}}, m_{\tilde{D}}, m_{\tilde{L}}, m_{\tilde{E}}\right\}=\left\{\frac{9}{10}, \frac{11}{10}, 1,1\right\} m_{\tilde{Q}}$ and $M_{1}=\frac{5}{3} M_{2} \tan ^{2} \theta_{W}$. The choice of a common trilinear coupling and the correlation between the soft sfermion masses are inspired by unification. For the plots in Fig. 通 we have taken: $\tan \beta=10, \mu=-100 \mathrm{GeV}, A=-500 \mathrm{GeV}, m_{\tilde{Q}}=300 \mathrm{GeV}, M_{A}=500 \mathrm{GeV}$, and $M_{2}=400 \mathrm{GeV}$. Choosing different sets of parameters, the basic characteristics of these plots remain indifferent. In fact, the soft gaugino masses $M_{1,2}$ affect only the Higgs sector through radiative corrections.

In Fig. 团 the relative correction $\Delta \sigma / \sigma_{0}$ is presented as a function of $\sqrt{s}$ for two different sets of parameters. The solid line corresponds to the set $\tan \beta=10, \mu=-100 \mathrm{GeV}$, $A=-500 \mathrm{GeV}, m_{\tilde{Q}}=300 \mathrm{GeV}, M_{A}=500 \mathrm{GeV}$, and $M_{2}=400 \mathrm{GeV}$, whereas for the dashed line we have taken $\tan \beta=40, \mu=-300 \mathrm{GeV}$ and $A=-100 \mathrm{GeV}$, keeping the rest of them unchanged. This figure shows that the size of the one-loop correction to the Higgs production cross section is practically constant for $\sqrt{s}>500 \mathrm{GeV}$ and weighs about $-15 \%$, almost independently of the choice of the SUSY parameters. The reason for this weak dependence is that the one-loop corrections are dominated by the fermion loops, and therefore the total correction is not very sensitive to the choice of the SUSY parameters. In this region, $\sqrt{s}>500 \mathrm{GeV}$, one can compare this constant correction with the effective approximation of Ref. [10], where one only corrects the $W W h^{0}$ coupling. Although the sign of this approximation is correct, it does not fully account for the whole effect.

In Fig. 0 we have plotted the cross section as a function of $m_{h}$ for the SM case, for $\sqrt{s}=0.8 \mathrm{TeV}$ (red lines) and $1 \mathrm{TeV}$ (black lines). The dashed lines correspond to 


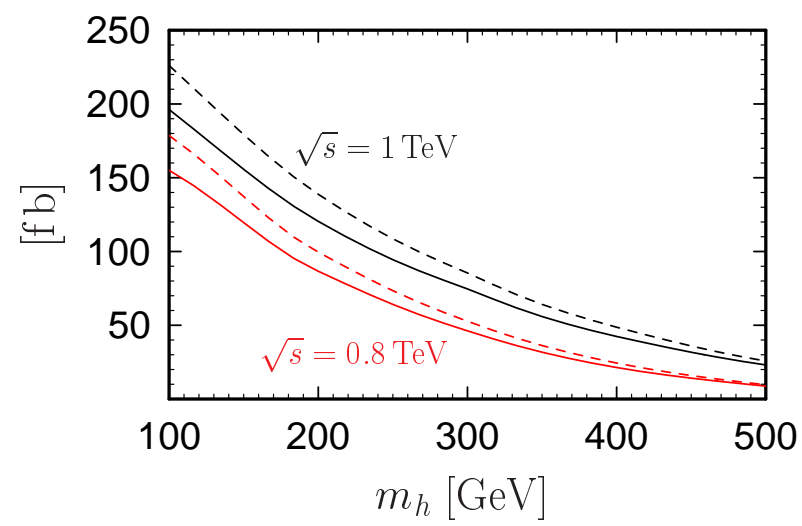

Figure 5: Cross sections for the SM case, for $\sqrt{s}=0.8 \mathrm{TeV}$ (red lines) and $1 \mathrm{TeV}$ (black lines). The dashed lines correspond to the tree-level cross section, whereas the solid lines to the one-loop corrected one.

the tree-level cross section for $e^{+} e^{-} \rightarrow \bar{\nu} \nu h^{0}$, whereas the solid lines contains the oneloop correction stemming from the fermion loops. In addition, the couplings have been adjusted to the SM corresponding couplings. The plot exhibits the expected dependence of the cross section on $m_{h}$. What must be noticed is that especially for small Higgs boson masses $\lesssim 200 \mathrm{GeV}$, the size of the fermion loops correction becomes important for the correct determination of the Higgs boson mass.

Finally, Fig. 6 exhibits the percentage of the sfermion loops to the total one-loop correction as a function of $\tan \beta$ (left) and $\mu$ (right), for two different values of $\mu$ and $\tan \beta$, respectively, as shown in the figure. In the left (right) figure we have chosen $A=-100 \mathrm{GeV}(A=-400 \mathrm{GeV})$. The rest of SUSY parameters are: $m_{\tilde{Q}}=300 \mathrm{GeV}$, $M_{A}=500 \mathrm{GeV}$, and $M_{2}=400 \mathrm{GeV}$. Here $\sqrt{s}$ has been fixed to $1 \mathrm{TeV}$. The grey area in the right figure is excluded due to the chargino mass bound. We see that the maximum value of order $10 \%$ can be achieved for large values of $\mu$ and $\tan \beta$. There, due to the significant mixing in the stop and sbottom sector, the contribution of stops and sbottoms in the loops is enhanced. For these values of SUSY parameters the sfermion masses approach their experimental lower bounds. But even there the dominant correction, at least $90 \%$ of the total correction, is due to the fermion loops.

In conclusion, we have calculated the fermion/sfermion loops corrections to the single Higgs boson production $e^{+} e^{-} \rightarrow \bar{\nu} \nu h^{0}$ in the context of the MSSM and SM. They are supposed to be the dominant radiative corrections. For energies relevant to the future linear colliders, $\sqrt{s} \gtrsim 500 \mathrm{GeV}$, the $W W$ fusion channel dominates the cross section. In general, the correction due to fermion/sfermion loops is negative and yields a correction 

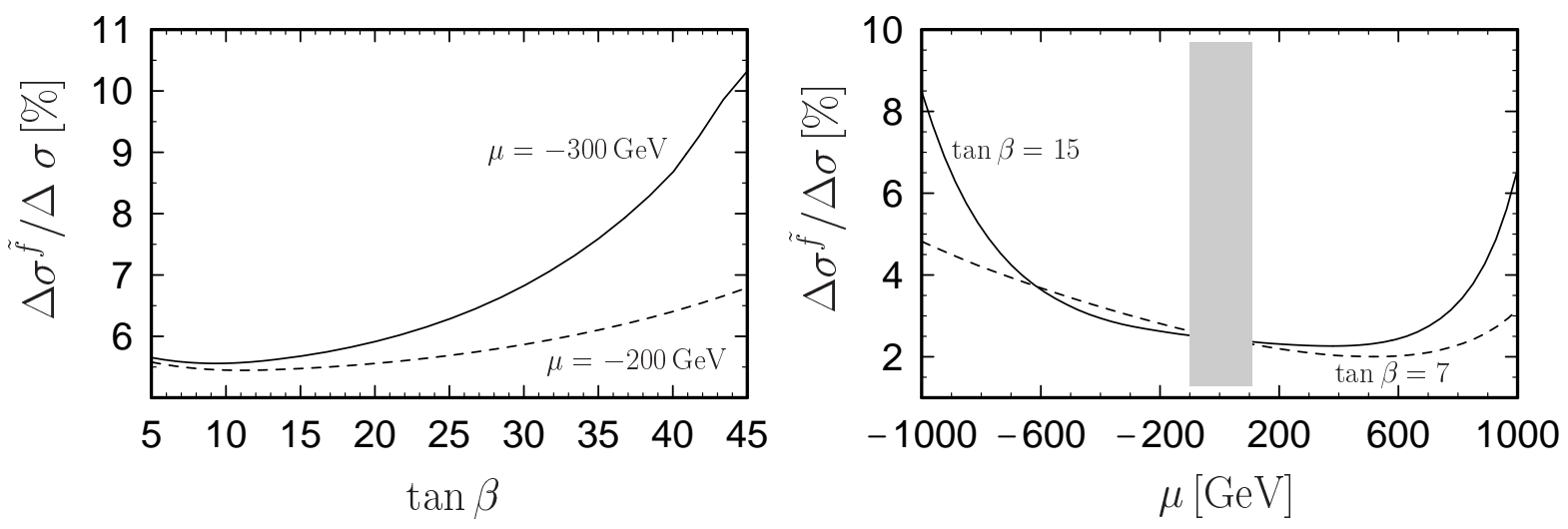

Figure 6: The percentage of the sfermions to the total one-loop correction as a function of $\tan \beta$ (left) and $\mu$ (right). The rest of the SUSY parameters have been fixed as described in the text. Here $\sqrt{s}=1 \mathrm{TeV}$. The grey area in the right figure is excluded due to the chargino mass bound.

to the cross section of the order of $-10 \%$. The bulk of this correction stems from the fermion loops, and usually turns to be more than $90 \%$ of the total correction. For the case of maximal mixing in the sfermion mass matrices, the contribution of the sfermion loops is enhanced, but nevertheless weighs less than $10 \%$ of the total one-loop correction. As the correction is dominated by fermion loops and is rather independent of $\sqrt{s}$ for $\sqrt{s}>500 \mathrm{GeV}$, we think that it can be approximated by a factor correction to the tree-level cross section. Such an approximation would be most useful for including initial state radiation (ISR) and beamstrahlung in an efficient way.

\section{Acknowledgements}

V. C. S. acknowledges support by a Marie Curie Fellowship of the EU programme IHP under contract HPMFCT-2000-00675. The authors acknowledge support from EU under the HPRN-CT-2000-00149 network programme. The work was also supported by the "Fonds zur Förderung der wissenschaftlichen Forschung" of Austria, project No. P13139PHY.

\section{References}


[1] LEP Collaborations, CERN-EP/2001-055 (2001), hep-ex/0107021; LEP Higgs Working Group, hep-ex/0107030, http://lephiggs .web.cern.ch/LEPHIGGS.

[2] D. R. T. Jones, S. T. Petcov, Phys. Lett. B84 (1979) 440; R. N. Cahn, S. Dawson, Phys. Lett. B136 (1984) 96; G. L. Kane, W. W. Repko, W. B. Rolnick, Phys. Lett. B148 (1984) 367; R. N. Cahn, Nucl. Phys. B255 (1985) 341; B. A. Kniehl, Z. Phys. C55 (1992) 605.

[3] G. Altarelli, B. Mele, F. Pitolli, Nucl. Phys. B287 (1987) 205.

[4] W. Kilian, M. Krämer, P. M. Zerwas, Phys. Lett. B373 (1996) 135.

[5] J. Ellis, M. K. Gaillard, D. V. Nanopoulos, Nucl. Phys. B106 (1976) 292; J. D. Bjorken, Proc. Summer Institute on Particle Physics, SLAC Report 198 (1976); B. W. Lee, C. Quigg, H. B. Thacker, Phys. Rev. D16 (1977) 1519; B. L. Ioffe, V. A. Khoze, Sov. J. Part. Nucl. 9 (1978) 50.

[6] D. Rainwater, D. Zeppenfeld, JHEP 12 (1997) 5; D. Rainwater, D. Zeppenfeld, K. Hagiwara, Phys. Rev. D59 (1999) 014037; T. Plehn, D. Rainwater, D. Zeppenfeld, Phys. Lett. B454 (1999) 297.

[7] Report of the Tevatron Higgs Working Group, M. Carena, J. S. Conway, H. E. Haber, J. D. Hobbs, FERMILAB-Conf. 00/279-T, hep-ph/0010338.

[8] Proceedings of the Large Hadron Collider Workshop, Aachen 1990, CERN 90-10, Vol. 1, Eds.: G. Jarlskog, D. Rein.

[9] B. A. Kniehl, Phys. Rep. 240 (1994) 211.

[10] B. A. Kniehl, Phys. Rev. D53 (1996) 6477.

[11] B. A. Kniehl, M. Steinhauser, Nucl. Phys. B454 (1995) 485.

[12] H. Eberl, W. Majerotto, V. C. Spanos, in preparation.

[13] H. Eberl, W. Majerotto, M. Kincel, Y. Yamada, Phys. Rev. D64 (2001) 115013; Nucl. Phys. B625 (2002) 372.

[14] M. Carena, M. Quiros, C. E. M. Wagner, Nucl. Phys. B461 (1996) 407. 